\title{
47. COLLISIONAL MODEL OF ASTEROIDS AND THEIR DEBRIS
}

\author{
J.S. Dohnanyi \\ (Bellcomm, Inc., Washington, D.C., U.S.A.)
}

\begin{abstract}
A BST RACT
A collisional model for interplanetary debris, formulated earlier by the writer, is applied presently to the problem of asteroids and their debris. Following observational evidence, a population-index type-number density function is assumed. The integro-differential equation describing the evolution of a system of colliding brittle particles derived earlier is satisfied for this special case. The result is a stable steady state solution subject to certain boundary conditions. It is found that catastrophic collisions dominate over the erosion process in the present steady state model.

Using a root mean-squared average collision velocity of $5 \mathrm{~km} / \mathrm{sec}$, the population index, $\alpha$, for asteroids is calculated to be $\alpha=1.837$. This value of $\alpha$ is rather insensitive to the physical parameters and is within the margin of error of an empirical least squares fit by the writer $\alpha=1.80 \pm .04$ to the distribution of asteroids reported by Kuiper et al. If the largest three asteroids are statistically interpreted, the distribution of asteroids in the asteroidal belt is consistent with the present collisional model. This would imply that most asteroids are fragments from some parent object(s).

The theoretical distribution is normalized to the observed number of asteroids in the survey by Kuiper $e t$ al., and using the present theory various statistical properties of the population of asteroids and their debris are calculated. The total mass of objects in the asteroidal belt is almost entirely concentrated in the masses of the three largest objects. The total asteroidal mass, crushed yearly, is of the order of $10^{12} \mathrm{~kg}$ and the rate of mass loss to radiation forces is about $7 \times 10^{10} \mathrm{~kg} / \mathrm{yr}$.

The mean lifetime of large asteroids is found to be of the order of $10^{8}-10^{9}$ years. The lifetime of objects is limited by the probability of catastrophic collisions; erosion and radiation damping only have a minor influence on the particle lifetimes.

The erosion rate of the largest objects is of the order of $1 \mathrm{~m} / 10^{6} \mathrm{yr}$, and this rate decreases for smaller objects. Comparison with erosion rates estimated by Whipple indicates that the lifetimes, with respect to erosion, of objects smaller than about $1 \mathrm{~kg}$ may be dominated by collisions with cometary particles.
\end{abstract}

\section{Introduction}

It is customary in the literature to describe the distribution of the masses of interplanetary particles by power-law functions. The number of particles in a mass range $m$ to $m+\mathrm{d} m$ is then taken to be $A m^{-\alpha} \mathrm{d} m$, where $A$ and $\alpha$ are constants; the latter is called the 'population index'. Observational evidence in support of such a special form for the number-density function has been advanced, among others, for radar meteors (Kaiser, 1961; Southworth, 1967), photographic meteors (Hawkins and Upton, 1958; Dohnanyi, 1966, 1967a), meteorites (Hawkins, 1960), and asteroids (Kuiper et al., 1958). In an effort to understand the physical significance of populationindex type-number density functions for interplanetary particles it has been undertaken by the writer to treat the dynamic interaction of these particles theoretically (Dohnanyi, 1967b, $c$ ); the present paper is an application of this previous work, to the 
distribution of asteroids and their debris. The physical model adopted is one where the interplanetary objects in the asteroidal belt undergo mutual inelastic collisions resulting in fragmentation. Since large asteroids are not believed to be constantly replenished, no external source function for particle injection is employed. Results of the analysis indicate that under simplifying but plausible assumptions such a system of particles does indeed evolve into a population index-type distribution (cf. Dohnanyi, $1967 b, c)$. The results are then applied to estimate the number density and other statistical properties of debris in the asteroidal belt.

\section{Observational Evidence}

This section is a discussion of the distribution of known asteroids together with some of their statistical physical properties. In their survey of asteroids, Kuiper et al.

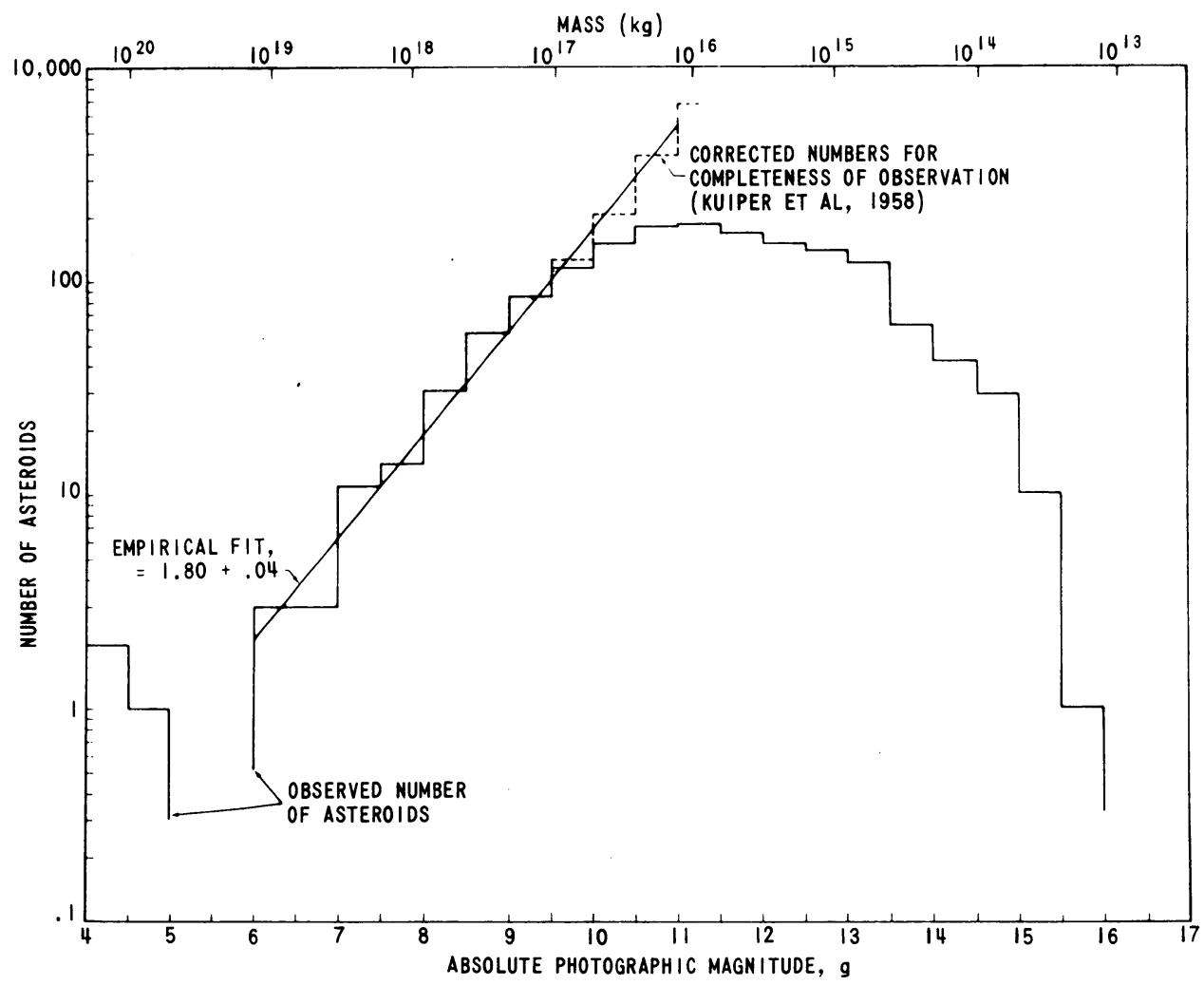

FIG. 1. Total number of observed asteroids (in half-magnitude intervals) as a function of absolute photographic magnitude (Kuiper et al., 1958). A mass scale, associated with the magnitude scale, is plotted at the top of the figure. Dashed histogram is the probable number of asteroids, based on observational selection. The sloped solid line is a least-squares fit to the data $(6<g<11)$ by the writer. 
(1958) have published the distribution of 1554 asteroids as a function of absolute photographic magnitude $g$ per half-magnitude interval.

The results of Kuiper et al. (1958) are plotted in Figure 1. The solid-line histogram is the number of asteroids in each half-magnitude interval as a function of $g$. A mass scale, based on a geometric albedo of $\cdot 2$ and density of $3 \cdot 5 \times 10^{3} \mathrm{~kg} / \mathrm{m}^{3}$, has been associated, by the writer, with the magnitude scale. The results are complete up to a limiting magnitude of $g \leqslant 9 \cdot 5$, i.e., the observed number of these objects is believed to equal the true number. Above $g \approx 9 \cdot 5$, the difference between the true and observed number of asteroids begins to increase due to selection effects. The dashed-line histogram is the probable number of asteroids using the 'completeness' factor of Kuiper et al. (1958). These authors have tabulated the maximum and minimum probable numbers of asteroids, and the dashed-line histogram in Figure 4 is the mean. We have plotted the estimated probable numbers up to the value where the correction factor, due to selection, is of the order of 2 . When the correction factor is much greater than 2, considerable uncertainties may be present.

It can be seen from the figure that a straight line (on this double logarithmic plot) is a good representation of the data for asteroids with $g$ greater than 6 . The solid straight line is a least-squares fit, by the writer, with the result

$$
f(m) \mathrm{d} m=\text { constant } \times m^{-1 \cdot 80 \pm \cdot 04} \mathrm{~d} m / \text { volume, }
$$

where $f(m) \mathrm{d} m$ is the number of asteroids in the small but arbitrary mass range from $m$ to $m+\mathrm{d} m \mathrm{~kg}$ per unit volume of space, 'volume' is the volume of the asteroidal belt which includes the orbits of the asteroids under discussion.

The number density function Equation (1) has the general form

$$
f(m) \mathrm{d} m=A m^{-\alpha} \mathrm{d} m,
$$

where $A$ is a constant equal to the number density of objects with unit mass, and $x$ is called the 'population index'.

\section{Collisional Model}

\section{A. IMPACT MECHANICS}

Interplanetary space contains a very large number of objects having different masses and orbits, which are believed to collide frequently with each other inelastically. When such a collision occurs at a sufficiently high relative velocity, fragmentation results. In the present study, the relative velocities will be comparable to those of particles in space traveling in different and sometimes intersecting orbits. The impact velocity will therefore be of the order of kilometers per second, which is sufficiently high to cause fragmentation. 
Following Dohnanyi (1967c), the mass distribution of fragments produced during impact will be assumed to have the form

$$
g\left(m ; M, M_{2}\right) \mathrm{d} m=(2-\eta) M_{\mathrm{e}}\left(M, M_{2}\right) M_{b}^{\eta-2}(m) m^{-\eta} \mathrm{d} m .
$$

Here, $g\left(m ; M, M_{2}\right) \mathrm{d} m$ is the number of particles having a mass between $m$ and $m+\mathrm{d} m$ produced during the impact of a mass $M$ with another, larger mass $M_{2} . M_{\mathrm{e}}$ is the total ejected mass and is a function of the colliding masses; $M_{\mathrm{b}}$ is the mass of the largest fragment and is taken to be a function of the smaller colliding mass $M . \eta$ is a constant, equal to $1 \cdot 8$, approximately. For erosive collisions $M_{\mathrm{e}}$ is taken to be (Dohnanyi, $1967 b, c)$

$$
M_{\mathrm{e}}=\Gamma M, \quad I^{\prime} M<M_{2},
$$

and for catastrophic collisions

$$
M_{\mathrm{e}}=M+M_{2}, \quad \Gamma^{\prime} M>M_{2} .
$$

The mass of the largest fragment is taken to be

$$
M_{b}=\Lambda M
$$

The quantities $\Lambda$ and $\Gamma^{\prime}$ are functions of the impact velocity; $\Gamma^{\prime}$ is the largest mass completely disrupted by a projectile of unit mass and is taken to be

$$
\Gamma^{\prime}=50 \Gamma \text {. }
$$

Numerical values for $\eta, \Lambda$ and $\Gamma$ are based on experimental results for basalt by Gault et al. (1965) for hypervelocity impact into semi-infinite targets (see Dohnanyi, $1967 b, c$, for a more detailed discussion).

\section{B. COLLISION EQUATION}

In this section the mathematical formulation of the evolution of a system of inelastically colliding particles is discussed. All objects will be assumed spherical and of identical material properties. Given that $f(m, t) \mathrm{d} m$ is the number of particles per unit volume having a mass between $m$ and $m+\mathrm{d} m$ at a time $t$, this function will change as a result of collisions between the particles because many new ones are constantly created (by fragmentation) and others destroyed. The system possesses a 'sink' in the sense that sufficiently small particles are removed by radiation effects. In what follows, the system will be assumed sufficiently random that an effective average collisional velocity is meaningful and the collision cross-section is taken as the cross-sectional area of the colliding spherical particles.

An equation defining the collective evolution of our system of particles can now be written. The time rate of change of the number of particles in a mass range of $m$ to 
$m+\mathrm{d} m$ is given, in a schematic form, by the following expression:

I

$\frac{\partial(m, t)}{\partial t} \mathrm{~d} m=$\begin{tabular}{l}
$\begin{array}{l}\text { rate of change of the number of particles per unit } \\
\text { volume and unit time in mass range } m \text { to } m+\mathrm{d} m \text { due } \\
\text { to erosion of these particles by collisions with smaller } \\
\text { ones. }\end{array}$ \\
\hline
\end{tabular}

II

\begin{tabular}{|l|l|} 
II \\
- $\quad \begin{array}{l}\text { rate of loss, because of 'catastrophic' collisions, in the } \\
\text { number of particles per unit volume and unit time in } \\
\text { the mass range } m \text { to } m+\mathrm{d} m\end{array}$ \\
\hline
\end{tabular}

III

number of particles in the mass range $m$ to $m+\mathrm{d} m$,
created per unit time and unit volume by erosive and
catastrophic collisional crushing of larger objects

Dohnanyi $(1967 b, c)$ discussed some of the properties of Equation (8). It was found that the collision equation (Equation (8)) is satisfied for a number-density function of the form Equation (2) provided that the population of particles has reached steady state (i.e., $\partial f / \partial t=0$ ) and that the following conditions are satisfied:

$$
\begin{gathered}
\frac{5}{3}<\alpha<2 \text { and } \\
\frac{1}{2}\left(\eta+\frac{5}{3}\right)<\alpha, \\
\Gamma^{\prime} \mu \ll M \ll \Lambda M_{\propto} / \Gamma^{\prime},
\end{gathered}
$$

where $\Gamma^{\prime} \mu$ is the largest mass that can be catastrophically disrupted by the smallest mass present, $\mu$, and $\Lambda M_{\infty} / \Gamma^{\prime}$ is the mass of the largest fragment when the largest mass in the population, $M_{\infty}$, is catastrophically disrupted by a collision with an object of mass $M_{\infty} / \Gamma^{\prime} . M_{\infty} / \Gamma^{\prime}$ is furthermore the mass of the smallest object that can catastrophically disrupt $M_{\infty}$.

We now consider the significance of the conditions, Equations (9), (10), and (11).

When $\alpha<\frac{5}{3}$, large objects are sufficiently abundant that their collisions will create so many fragments (distributed according to Equation (3)) that the particle creation rate (term II in Equation (8)) will dominate over other processes causing an evolution in the population of particles. When $\alpha>2$, erosion by small particles dominates the 
right-hand side of Equation (8), again causing the population to be time-dependent. In order to discuss the significance of Equation (10), one can note from Equation (3) that during a given collision the relative number of small fragments produced increases with $\eta$. When $\eta>2 \alpha-\frac{5}{3}$, the number of small fragments is so large (compared to the number of large fragments) that their creation rate exceeds their destruction rate and the population of small fragments increases with time. The steady state condition is thereby violated.

The third condition, Equation (11), states that one can only have population-index type of a distribution for masses in a range sufficiently far away from the end points $\mu$ and $M_{\infty}$. For masses $m \leqslant \Gamma^{\prime} \mu$ all collisions are catastrophic and for masses sufficiently close to $M_{\infty}$, the particle creation term (term III in Equation (8)) falls off.

Furthermore, the number of particles with masses $m<\mu$ and $M>M_{\infty}$ is zero and a separate mathematical treatment of Equation (8) near these end points is necessary before the form of the distribution in these regions can be established.

When a population-index type-number density function (Equation (2)) is substituted into the right-hand side of Equation (8), the various rate processes can be evaluated for given values of the physical parameters $\Lambda, \Gamma, \Gamma^{\prime}$, and $\eta$. Since $\Lambda \Gamma$ and $\Gamma^{\prime}$ are determined by the collision velocity $V$, the independent variables that determine the physical parameters are $V$ and $\eta$.

Figures 2 and 3 are plots of the numerical values of the various terms on the righthand side of Equation (8) vs. the population index $\alpha$ for two different sets of physical parameters. Since over the range of $\alpha$ chosen in the plot, the various terms have identical functional dependences on the particle mass $m$, we chose to plot the various terms in units of $K A^{2} m^{-2 \alpha+5 / 3}$. Here $A$ is the normalization constant appearing in Equation (2) and $K$ is given by

$$
K=V\left[3 \pi^{1 / 2} / 4 \rho\right]^{2 / 3},
$$

where $V$ is the impact velocity and $\rho$ is the material density of the colliding masses.

It can be seen from Figures 2 and 3 that steady state is reached for a value of $\alpha$ in the neighborhood of $\alpha=1.83$ to 1.84 . At this value of $\alpha$, the sum of all the particle creation and removal rates $\Sigma$, i.e. $\partial f / \partial t$, is zero. For lower values of $\alpha$ the particle creation rate $\psi$ (i.e. term III in Equation (8)) dominates and for larger values of $\alpha$ the particle-removal rate $\phi$ and $\theta$ (terms I and II in Equation (2)) dominate. The erosive particle-removal rate $\theta$ is quite small for $\alpha$ much less than 2 . This is due to the fact that the mass redistributed by an erosive collision is of the order of $\Gamma$ times the 'projectile' mass, but in a catastrophic collision the amount of mass redistributed may be as great as $\Gamma^{\prime}=50 \Gamma$ times the projectile mass. For values of $\alpha$ significantly lower than 2 , it can be shown (Dohnanyi, in preparation) that the erosive process $\theta$ is about $\Gamma / \Gamma^{\prime}=1 / 50$ times smaller than is the catastrophic collision process $\phi$. Furthermore, the contribution to the creation term $\psi$ of erosive collisions is of the order of $\Gamma / \Gamma^{\prime}=$ $1 / 50$ times the contribution of catastrophic processes. It is therefore inferred that a 
steady-state population of the type considered here (i.e. $\Gamma \ll \Gamma^{\prime}$ ) is maintained mainly by a balance of catastrophic processes. It can furthermore be concluded (Dohnanyi, in preparation) that a first-order solution of Equation (8) for $\alpha$ under the condition $\Gamma \ll \Gamma^{\prime}$ yields

$$
\alpha=11 / 6 .
$$

This result (Equation (12)) is independent, in first order, of the numerical values of the physical parameters, provided that $\Gamma^{\prime} \gg \Gamma$ and that the conditions Equations (9), (10) and (11) are satisfied. The relation, $\alpha=11 / 6$ is solely determined by the balance

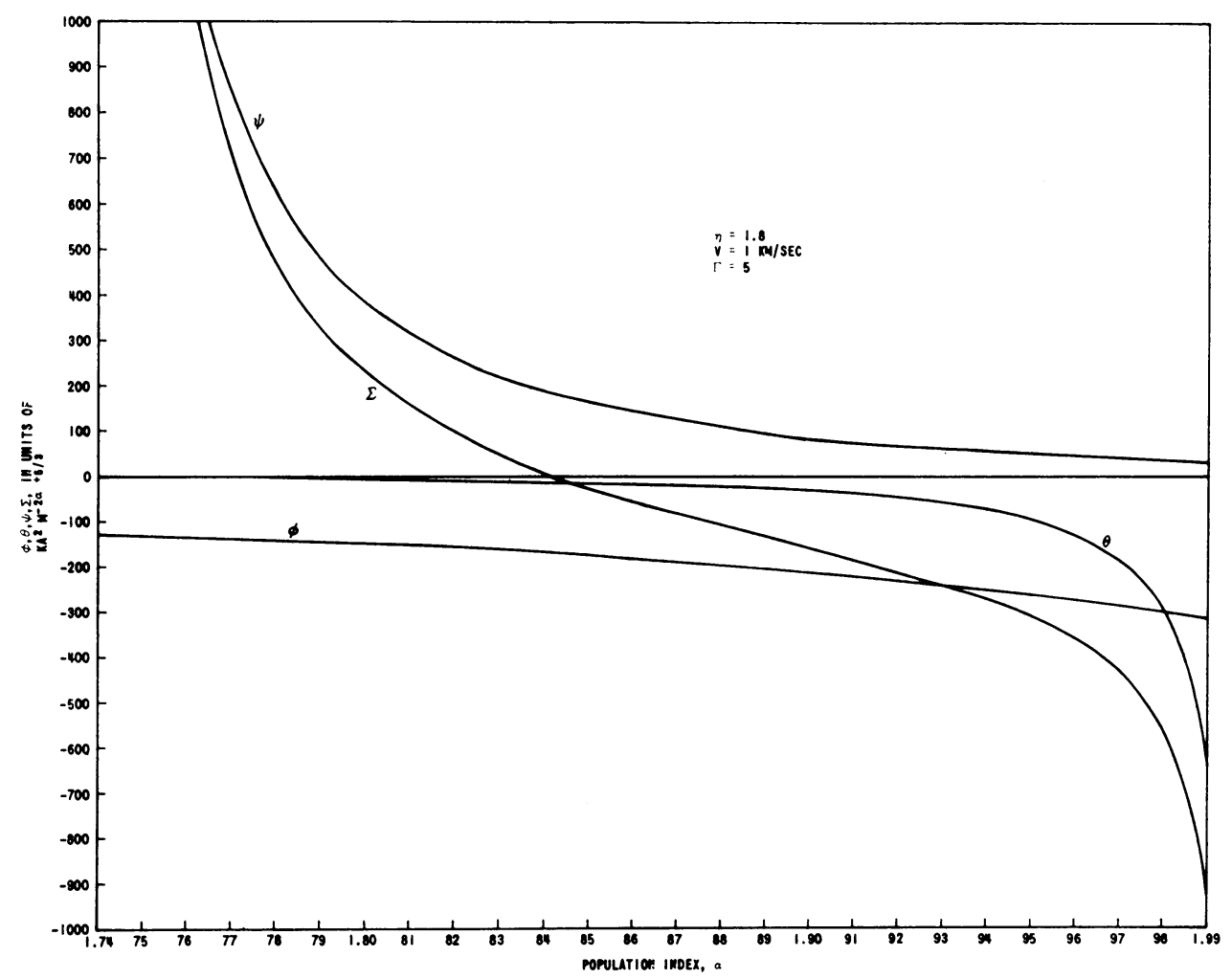

FIG. 2. Rate of change of the number of particles in units of $K A^{2} M^{-2 \alpha+5 / 3}$ per unit time and unit mass range as a function of the population index $\alpha . \psi=$ rate of change in the number of particles because of particle creation by fragmentation of larger objects, $\phi=$ rate of change in the number of particles because of catastrophic collisions, $\theta=$ rate of change in the number of particles because of erosion, $\Sigma=\psi+\phi+\theta$.

between the rate at which objects are removed from a given mass range by the catastrophic process and the rate at which they are 'replaced' by fragments produced during catastrophic encounters between larger masses. Numerical work indicates that the influence of higher-order terms on Equation (12) is of the order of $1 \%$. 


\section{Application and Discussion}

\section{A. MODEL DISTRIBUTION}

In this section, a model distribution of debris in the asteroidal belt is defined and compared with observation. This is accomplished by a suitable choice of the physical parameters and the number-density function is then normalized to the observed number of asteroids.

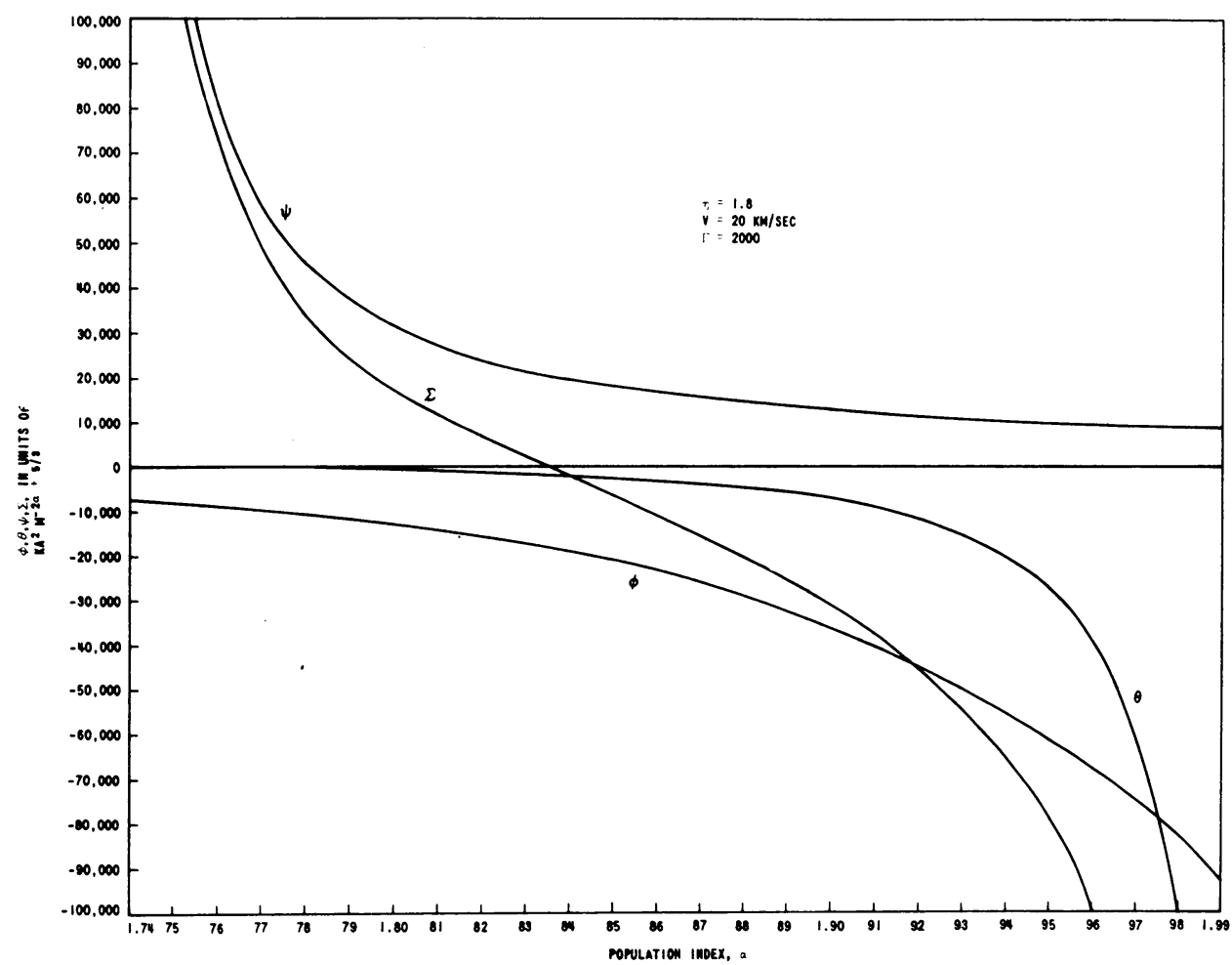

FIG. 3. Rate of change of the number of particles in units of $K A^{2} M^{-2 \alpha+5 / 3}$ per unit time and unit mass range as a function of the population index $\alpha . \psi=$ rate of change in the number of particles because of particle creation by fragmentation of larger objects, $\phi=$ rate of change in the number of particles because of catastrophic collisions, $\theta=$ rate of change in the number of particles because of erosion, $\Sigma=\psi+\phi+\theta$.

For asteroids we choose (Dohnanyi, in preparation) an average collisional velocity $V$ of $5 \mathrm{~km} / \mathrm{sec}$, a material specific gravity of $3 \cdot 5$, a population index for the comminution law $\eta=1 \cdot 8$, and a population index $*$ for the population of particles $\alpha=1 \cdot 837$. The normalization constant is so chosen that $81 \%$ of the asteroids are assumed to have an inclination of less than $10^{\circ}$ (Narin, 1966) and confined to a distance of $2 \cdot 2$ to $3 \cdot 5$

* This is determined by $V=5 \mathrm{~km} / \mathrm{sec}$ and $\eta=1 \cdot 8$. 
AU from the Sun. The result, for the model distribution, is

$$
f(m) \mathrm{d} m=2.5 \times 10^{-19} m^{-1 \cdot 837} \mathrm{dm} / \text { meter }^{3},
$$

where $f(m) \mathrm{d} m$ is the number density of asteroids in the asteroidal belt per $\mathrm{m}^{3}$ in the mass range of $m$ to $m+\mathrm{d} m \mathrm{~kg}$.

We now consider again the distribution of the known asteroids as given by Kuiper et al. 1958 (cf. Figure 1). Figure 4 is a repetition of Figure 1 with the theoretical results included for comparison. The theoretical population index $\alpha=1.837$ is seen

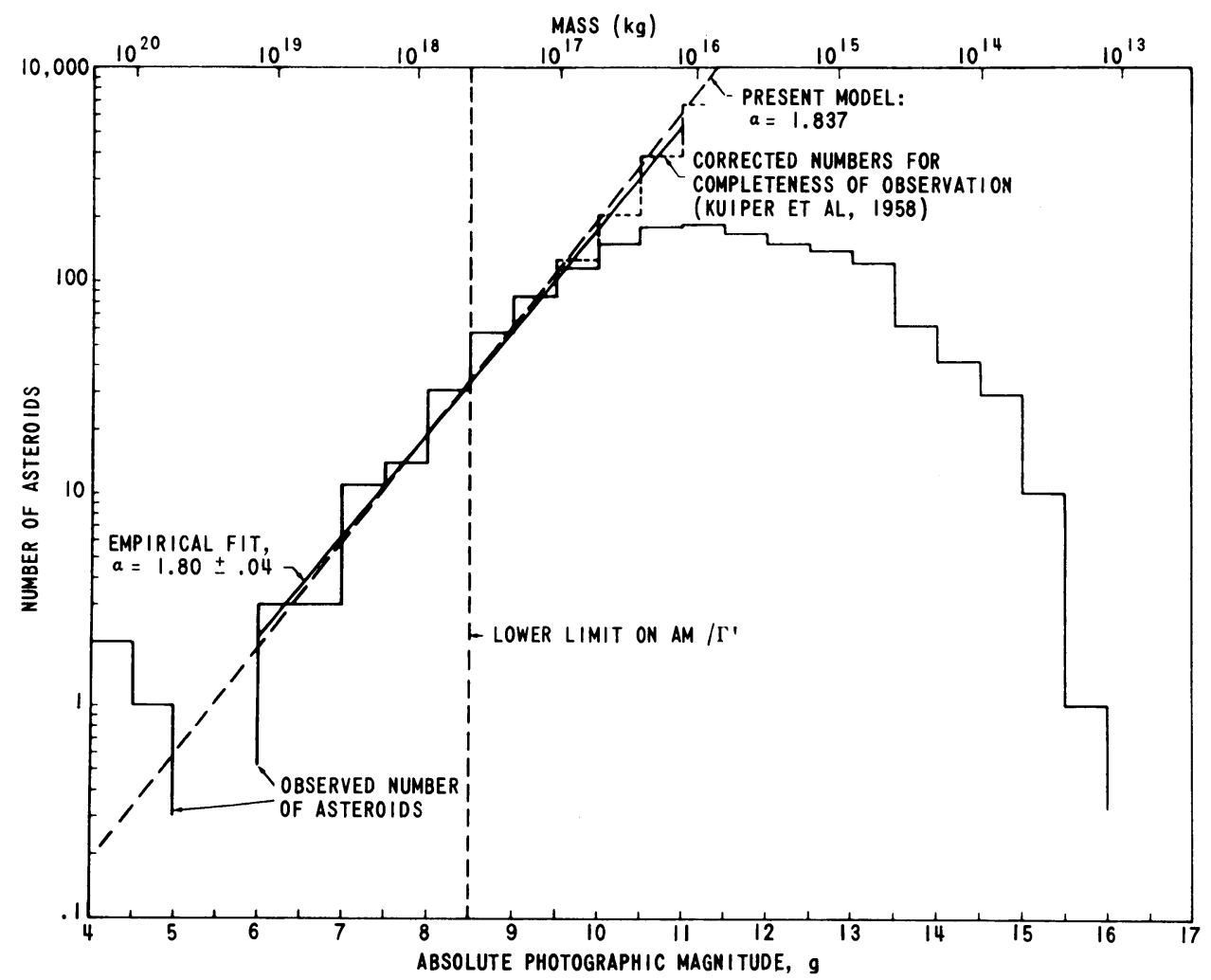

FIG. 4. Total number of observed asteroids (in half-magnitude intervals) as a function of absolute photographic magnitude (Kuiper et al., 1958). A mass scale, associated with the magnitude scale is plotted at the top of the figure. Dashed histogram is the probable number of asteroids, based on observational selection. The solid line is a least-squares fit to the data $(6<g<11)$ by the writer and the dashed straight line is the theoretical result discussed in the text.

to be within the margin of error of the empirical result $\alpha=1 \cdot 80 \pm \cdot 04$. The dashed vertical line is the value of $\Lambda M_{\infty} / \Gamma^{\prime}$ (cf. discussion following Equation(11)) on the basis of letting $M_{\infty}=1.7 \times 10^{22} \mathrm{~kg}$, which corresponds to $g=4$.

This choice for $M_{\propto}$ is, however, an extremely conservative lower limit. The number 
density of observed asteroids in the neighborhood of $g=4$ is (in a statistical sense) an order of magnitude higher than is the theoretical value (cf. Figure 4). If one divides the three largest asteroids into a fractional number of objects in the range of magnitudes $6>g>g_{\infty}$, where $g_{\infty}$ corresponds to a fictitious but statistically meaningful object, such that the theoretical result would predict cumulative mass of objects with $g<6$ equal to the mass of the three largest objects, the result is $M_{\infty}=$ order of $10^{22} \mathrm{~kg}$. Thus, $M_{\infty}$ increases by two orders of magnitude compared with our conservative estimate and $\Lambda M_{\infty} / \Gamma^{\prime}$ becomes of the order of $10^{20} \mathrm{~kg}$. These parameters would imply that the creation rate equals the destruction rate for asteroids having masses of about $10^{19}-10^{20} \mathrm{~kg}$ or smaller. It therefore follows that, if $M_{\infty}$ is defined in a statistical sense, the entire distribution of observed asteroids save the few largest ones has reached steady-state conditions and the creation rate equals the removal rate. Physically this means that the three largest objects act as a mass reservoir which feeds the population of smaller objects.

\section{B. STATISTICAL PROPERTIES OF ASTEROIDAL FRAGMENTS}

In this section, a number of statistical properties of the distribution of asteroids and their fragments are derived using the present collisional model, and their significance discussed.

Using Equation (13), one can calculate the rate at which particles are undergoing collisional processes described by the model. The result is Figure 5, where these rates are plotted logarithmically as a function of mass in $\mathrm{kg}$. Rates are expressed per year and are multiplied by the effective volume of the asteroidal belt, so that the result is the total number of objects undergoing the various processes in the asteroidal belt (rather than per unit volume) within a latitude of $\pm 10^{\circ}$.

$\psi(m) \mathrm{d} m$ is the total number of objects created per year in the mass range $m$ to $m+\mathrm{d} m \mathrm{~kg}$. We plot $\psi(m)=\psi(m) \mathrm{d} m / \mathrm{d} m$ rather than $\psi(m) \mathrm{d} m$, so that the yearly rate corresponding to a certain value of $m$ has to be multiplied by a desired mass range $\mathrm{d} m$ in order to obtain the number of objects yearly created in that mass range. We find, e.g., that for $m=10^{8} \mathrm{~kg}, \psi=10^{-6} / \mathrm{yr} \mathrm{kg}$. This means that if we take a mass range of $10^{8}$ to $10^{8}+10^{7} \mathrm{~kg}$, the yearly creation rate, in this range, becomes $\psi \times 10^{7}$ $\mathrm{kg} \simeq 10 / \mathrm{yr}$ and hence, ten objects in this mass range are created every year (on the average) by collisional break-up of larger objects. The departure from linearity of $\psi(m)$ at $m \simeq 10^{15} \mathrm{~kg}$ is caused by the fact that one is approaching $M_{\infty}$, the top mass of the distribution, if the latter is conservatively defined.

The quantity $\phi(m) \mathrm{d} m$ is the total number of objects in the mass range $m$ to $m+\mathrm{d} m$ destroyed by catastrophic collisions per year and expressed in appropriate units. The numerical values of $\psi(m)$ and $\phi(m)$ are almost equal and are plotted in Figure 5 as a single curve for masses less than $10^{15} \mathrm{~kg}$; this reflects the relative unimportance of the erosion process in the steady-state distribution described by the present model. 
The expression $\dot{M}(m)$ is the total mass in $\mathrm{kg}$ of objects having a mass of $m \mathrm{~kg}$ or smaller created yearly due to collisional fragmentation. It can be seen from Figure 5 that the total asteroidal mass, crushed yearly, is about $10^{12} \mathrm{~kg}$.

For $m$ sufficiently small, $\dot{M}(m)$ gives an estimate of the mass removed yearly from

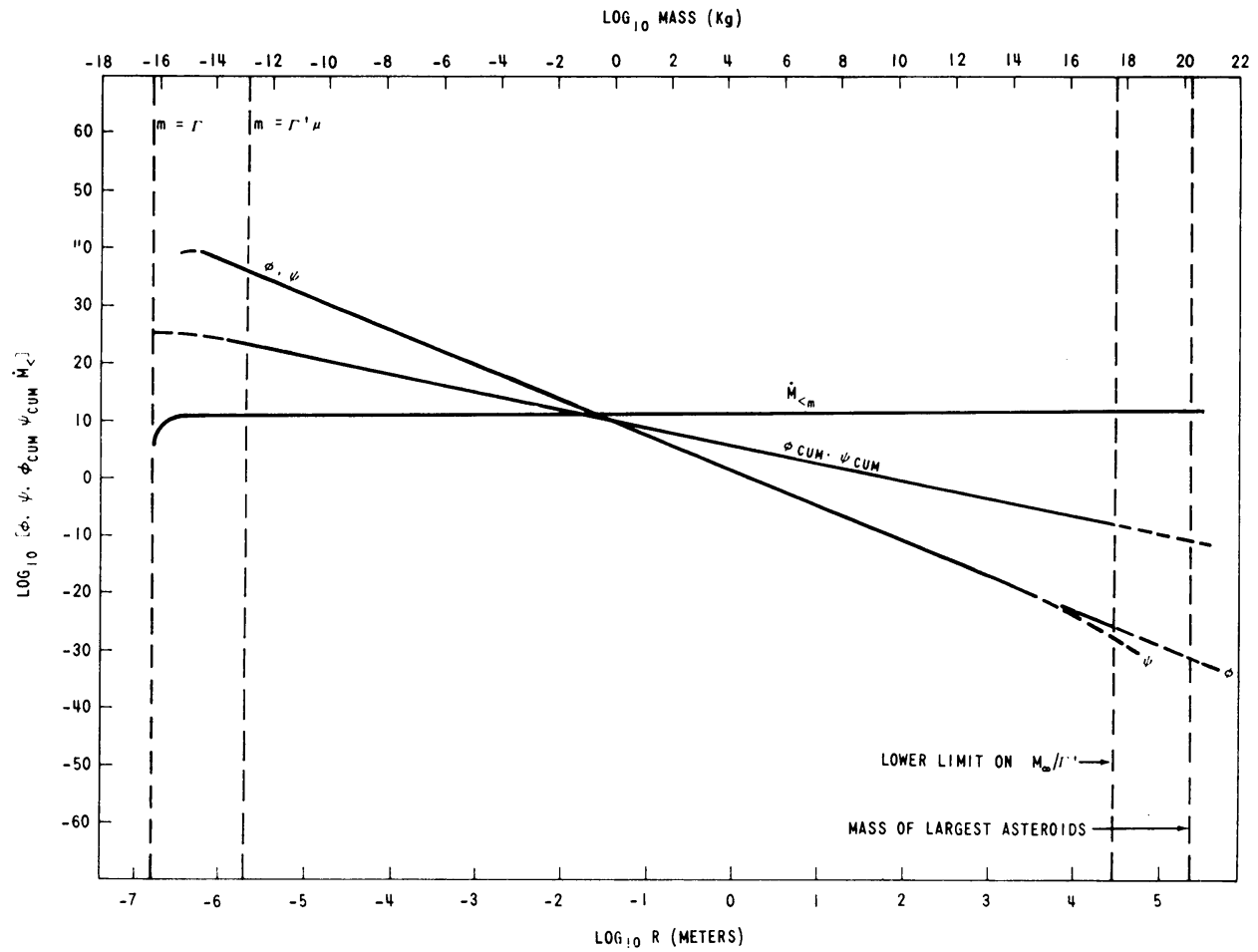

FIG. 5. Double logarithmic plot as a function of particle mass (or particle radius) of the following yearly rates; $\phi, \psi$ particle removal rate per unit mass due to catastrophic collisions and particle-creation rate per unit mass due to fragmentation, respectively. $\dot{M}_{<m}$, comulative mass of particles with a mass $m$ or smaller, created annually by fragmentation.

the asteroidal belt by radiation forces. Since in this model we have arbitrarily chosen $\mu$ as the smallest object created, $\dot{M}(\mu)=0$. It is, however, to be expected that objects with a mass $\Gamma^{\prime} \mu$ or less will be strongly influenced by radiation forces since they are no longer large enough to experience collisional processes by much smaller particles, the latter being absent because they are blown away by radiation pressure. We therefore assume, somewhat arbitrarily, that an upper limit of the yearly mass loss from the asteroidal belt due to radiation damping (Robertson, 1936) and radiation pressure is given by $\dot{M}\left(\Gamma^{\prime} \mu\right)$ in $\mathrm{kg} / \mathrm{yr}$. According to Figure 5 , this quantity is about $7 \times 10^{10} \mathrm{~kg} / \mathrm{yr}$ (about 70 million tons/yr) and is probably quite enough to maintain the zodiacal cloud. In view, however, of the fact that the distribution of such small particles is 
likely to be strongly influenced by collisions with cometary meteoroids, a reliable figure for the yearly mass loss can only be given after the problem under discussion has been treated.

We note that when $\dot{M}\left(\Gamma^{\prime} \mu\right)$ is averaged over a period of $10^{9}$ years, the result is $7 \times 10^{19} \mathrm{~kg}$, which is the same order of magnitude as the mass of one of the largest objects present. This mass-removal rate therefore requires the presence of one parent object in addition to others already available in very early times and therefore does not involve arbitrary assumptions.

We now consider the total mass $M_{T}$ of the asteroids and their debris within a latitude of $10^{\circ}$. Using Equation (13) we obtain

$$
M_{T}=\int_{\mu}^{M_{\alpha}} f(m) \mathrm{d} m \times \text { volume }=3 \times 10^{20} \mathrm{~kg},
$$

where the conservative value for $M_{\propto}$ has been used.

It can be seen from Figure 4 that $3 \times 10^{21} \mathrm{~kg}$ is the same order of magnitude as the

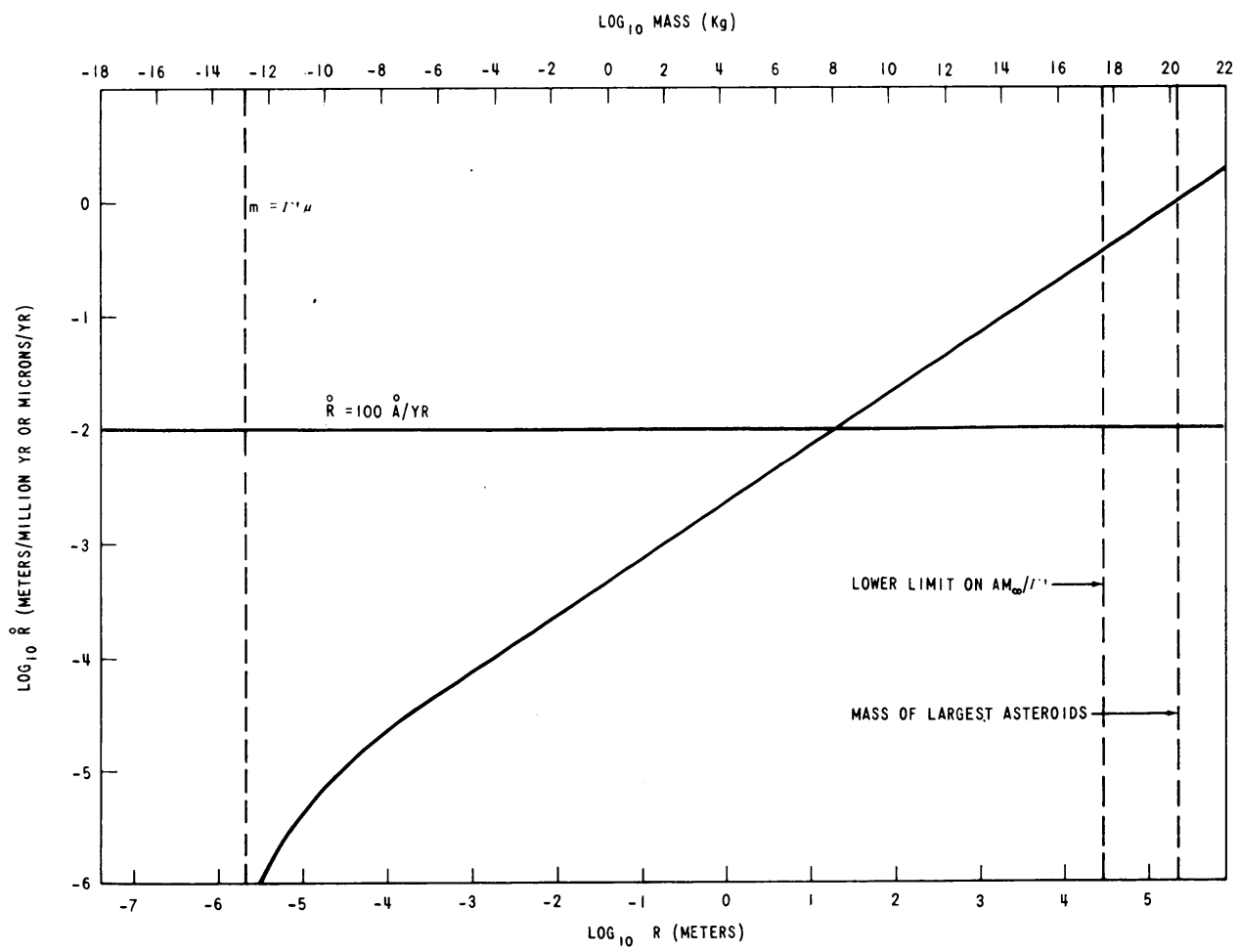

FIG. 6. Statistical rate of change because of erosion of the particle radius in meters per $10^{6}$ years (or microns per year) as a function of particle mass (or particle radius). The dashed horizontal line corresponds to a linear erosion rate of $100 \AA / y r$. 
mass of one of three large asteroids. We therefore conclude that the largest asteroids contain practically all the mass in the asteroidal belt.

Figure 6 is a double logarithmic plot of the rate $\dot{R}$ at which the radius of a spherical object changes with time due to erosion as a function of the mass in $\mathrm{kg}$ of the object; the radius of an object having a mass $m$ and a density of $3.5 \times 10^{3} \mathrm{~kg} / \mathrm{m}^{3}$ is also indicated. The mathematical expression for $\dot{R}$ is readily derived; the result is

$$
\dot{R}=-5.15 \times 10^{-4}\left[m^{\cdot 163}-\left(\Gamma^{\prime} \mu\right)^{\cdot 163}\right],
$$

where $\dot{R}$ is expressed in meters per $10^{6}$ years.

The most obvious feature of Equation (14) (cf. Figure 5) is that $\dot{R}$ is not a constant but is a function of the mass of the object undergoing erosion. We remind the reader that the process of erosion as defined in this paper is not due alone to small micronsized particles but is due to the dominating process of collisions with all masses up to the range of $m / \Gamma^{\prime}$, where $m$ is the mass of the objects being eroded. Since in our model the population index $\alpha$ is less than 2, the particle-number density is such that the total mass eroded away from a given object by collisions with microparticles is much less than is the mass eroded away by larger objects. We therefore have a situation where objects that are large in comparison with microparticles are sufficiently abundant to dominate the erosion process. The erosion rate of an object with a given mass $m$ is then determined by the abundance of all objects with masses less than $m / \Gamma^{\prime}$ which is the mass required to produce catastrophical break-up of $m$ and hence the strong non-linear dependence of $\dot{R}$ on $m$.

It can be seen from Figure 6 that $\dot{R}$ of a particle increases with increasing particle mass. For large asteroids having a mass of $10^{18} \mathrm{~kg}$, the erosion rate is of the order of $1 \mathrm{~m} / 10^{6} \mathrm{yr}$ which is equal to $\alpha \mathrm{km}$ in $10^{9}$ years. There is no way to check the accuracy of this figure, but we note that the lunar highlands are saturated with craters of a size range of tens of kilometers and smaller. Assuming most of these craters to be of impact origin and that the highlands have an age of the order of billions of years (and the maria are much younger), we note that if the Moon did not possess a gravitational field it would surely be 'eroded' to a depth of several kilometers. In view of the fact that the impact environment of the Moon has been less severe than is the environment in the asteroidal belt, at least for a very long time in the past, our result in Figure 5 appears to be reasonable.

The values of $\dot{R}$ for small masses in Figure 5 are not realistic since no attention was given to the influence of erosion by cometary meteoroids and spallation due to cosmic rays. These processes have been estimated by Whipple (1953) to give rise to an erosion rate not exceeding about $100 \AA / \mathrm{yr}$ or $10^{-2} \mathrm{~m} / 10^{6} \mathrm{yr}$. This upper limit is indicated in Figure 5 as a horizontal dashed line. While Whipple's estimate applies to objects with orbits intersecting the Earth's orbit, his upper limit is still meaningful for particles in the asteroidal belt if the erosive effect of cometary meteoroids in the asteroidal belt is taken to be comparable to or lower than is the case near Earth. 
Figure 7 is a double logarithmic plot of particle lifetimes in years as a function of particle masses in $\mathrm{kg}$, as given by the present model. The lifetime of an object with respect to catastrophic collisions, $\tau_{\mathrm{cc}}$, is taken as the inverse of the probability that the object will experience a catastrophic collision and is given by

$$
\tau_{\mathrm{cc}}=2.46 \times 10^{6} \mathrm{~m}^{\cdot 17} \mathrm{yr},
$$

which is an upper limit since during a time $\tau_{\mathrm{cc}}$ the mass of the object will continuously erode into a smaller value and hence will have a smaller lifetime with respect to catastrophic collisions, because $\tau_{\mathrm{cc}}$ decreases with mass, as indicated by Equation(15). This

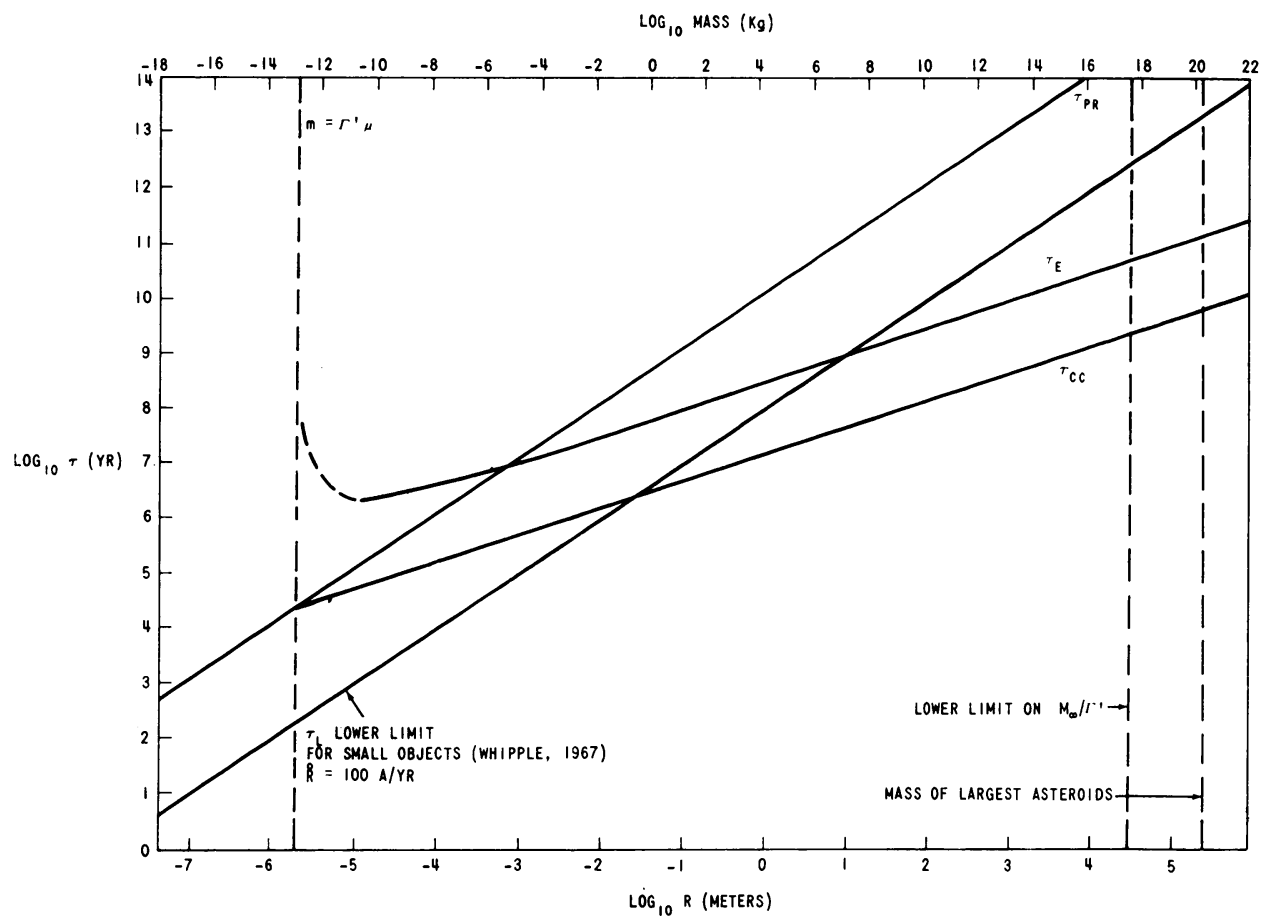

FIG. 7. Double logarithmic plot of particle lifetimes in years as a function of particle masses in $\mathrm{kg}$ (or particle radii in meters).

reflects the fact that, in the present model, the number of particles that can cause a disruptive encounter (for a given object) increases faster with decreasing particle mass than is the corresponding decrease in the collision cross-section. The value of $\tau_{\mathrm{cc}}$ for large asteroids is of the order of $10^{8}-10^{9}$ years (cf. Figure 7). Öpik (1963) has studied the lifetime of the Apollo asteroids with respect to planetary encounters, and it is interesting to note that he has derived lifetimes for these objects of similar order of magnitude $\left(\sim 10^{8} \mathrm{yr}\right)$ as our estimate of $\tau_{\mathrm{cc}}$ for large asteroids in the asteroidal belt. 
The lifetime with respect to erosion $\tau_{\mathrm{E}}$, is taken here somewhat arbitrarily, as the time for an object to erode to one half of its initial radius. A population index $\alpha=11 / 6$ $=1.8333$ has been used to facilitate integration. $\tau_{\mathrm{E}}$ is expressed in years and $m$ in $\mathrm{kg}$. The definition of $\tau_{\mathrm{E}}$ is seen therefore to be different from that of $\tau_{\mathrm{cc}}$ since the latter is the inverse probability of complete destruction and represents, therefore, an effective lifetime.

We also plot, in Figure 7, the particle lifetimes with respect to the PoyntingRobertson effect $\tau_{\mathrm{PR}}$ and the lower limit of the lifetime of small objects $\tau_{\mathbf{L}}$ due to the influence of cometary meteoroids and cosmic rays estimated by Whipple (1963). $\tau_{\mathrm{PR}}$ is taken here as the time required for an object to traverse radially one half of the asteroidal belt, because of radiation damping. It can be seen, from the figure, that for particles greater than about $10^{-7} \mathrm{~kg}$ (or $0.1 \mathrm{~mm}$ in radius) the process of catastrophic collision dominates the lifetime of the particles. Smaller particles may be subject to erosion by cometary particles to an extent that this latter mechanism dominates. $\tau_{\mathrm{E}}$ and $\tau_{\mathrm{PR}}$ are seen to be insignificant for all particles of reasonable size by comparison with $\tau_{\mathrm{cc}}$. It can furthermore be seen that for masses smaller than approximately $10^{-9} \mathrm{~kg}$ (or particle radius of about 30 microns) $\tau_{\mathrm{PR}}$ becomes shorter than $\tau_{\mathrm{E}}$. Since $\tau_{\mathrm{PR}} \gg \tau \mathrm{cc}$ over the entire range of particle masses, we see that our neglect of radiation damping in the collision equation, Equation (13), is justified.

It can also be seen from Figure 7 that the lifetime of the largest objects is of the order of $10^{9} \mathrm{yr}$. Some of the large objects may therefore have escaped catastrophic collisions in the past, but most others have not. For small objects, having a mass of the order of perhaps $10^{-5} \mathrm{~kg}$ or smaller, the influence of collisions with cometary meteoroids must be treated, before meaningful lifetimes for these particles can be estimated, and therefore the significance of the curves in Figure 7 for small masses is doubtful.

\section{Conclusion}

A collisional model of interplanetary particles is applied to the distribution of asteroids and their debris. An equation describing the collective dynamical interaction of these particles caused by inelastic collisions and fragmentation, derived by the writer, has been solved for the particle-number density function $f(m) \mathrm{d} m$ for the special case where the distribution has reached steady state conditions. The result is

$$
f(m) \mathrm{d} m=A m^{-\alpha} \mathrm{d} m .
$$

The population index $\alpha$ equals $11 / 6$ in a first approximation; higher-order terms contribute only slightly to $\alpha$. The value of $\alpha$ is remarkably insensitive to the values of the physical parameters.

This solution can be shown to be stable in the sense that if $\alpha$ is altered, an imbalance between the particle creation and removal rates is introduced which will cause the population index to return to its previous steady-state value. It can furthermore be 
shown (cf. Figures 2 and 3) that for steady-state conditions without an external source, the erosion rates have only a minor influence on the population when compared with the rates of catastrophic collisions and particle creation by fragmentation.

The results are then applied to the distribution of asteroids and their debris. The theoretical number-density function for asteroids is

$$
f(m) \mathrm{d} m=2.48 \times 10^{-19} m^{-1.837} \mathrm{~d} m / m^{3},
$$

where the normalization constant is based on observation and the population index 1.837 corresponds to steady-state conditions with a root mean-square collisional velocity of $5 \mathrm{~km} / \mathrm{sec}$.

A least-squares fit to the distribution of asteroids (Figure 4) catalogued by Kuiper et al. (1958) yields an empirical value for $\alpha$ of $1 \cdot 80 \pm .04$. The theoretical value of $\alpha$ is therefore seen to be within the margin of error of the empirical one.

Since the top masses are not replenished the distribution (Figure 4) assumes steadystate condition for asteroids having less than a given mass only (cf. Figure 5). The approximate value of this mass depends on our choice for $M_{\infty}$. The most conservative choice of $M_{\infty}$ is $1.88 \times 10^{20} \mathrm{~kg}$ corresponding to absolute photographic magnitude $g=4$ in Figure 4; this implies steady-state conditions for masses less than $10^{15} \mathrm{~kg}$. Since the largest three observed asteroids cluster, they can be redistributed artificially according to the theoretical distribution, Equation (15), while keeping the total mass invariant. Such a procedure is statistically permissible and represents the physical influence of the three top masses on the dynamics of the population of smaller objects more correctly than.would be the case if we disregarded them. The result is that practically all but the three largest asteroids have reached steady-state conditions. The three largest asteroids behave as a constant source of particles replenishing the distribution of large asteroids. This conclusion is also supported by the very close agreement between the theoretical and the observed population index of asteroids.

Using Equations (15), a number of useful statistical properties of asteroids can be calculated. The yearly total of asteroidal mass crushed is about $10^{12} \mathrm{~kg} / \mathrm{yr}$ (Figure 5). The amount of mass lost yearly from the asteroidal belt is about $7 \times 10^{10} \mathrm{~kg}$, but this figure may not be reliable because the influence on the population of small particles by cometary meteoroids has not been considered.

The total mass of asteroids and their debris is about $3 \times 10^{20} \mathrm{~kg}$, where the three heaviest objects, of this same order of magnitude, have not been properly included. When the top masses are considered, the total mass is of the order of $10^{21} \mathrm{~kg}$; it therefore follows that almost all of the mass in the asteroidal belt is concentrated in the three heaviest objects.

The erosion rate of an object in the asteroidal belt (Figure 6) is non-linear. The rate of change in the effective radius of the largest objects is about $1 \mathrm{~m} / 10^{6} \mathrm{yr}$. This rate decreases for smaller objects and for masses of about $10^{8} \mathrm{~kg}$ the rate equals $10^{-2}$ 
$\mathrm{m} / 10^{6} \mathrm{yr}$ which is the upper limit caused by erosion due to cometary particles and cosmic rays obtained by Whipple (1963).

The particle lifetimes with respect to catastrophic collisions $\tau_{\mathrm{cc}}$, erosion $\tau_{\mathrm{E}}$ and radiation damping $\tau_{\mathrm{PR}}$ have been calculated (Figure 7). $\tau_{\mathrm{cc}}$ is also very much shorter than $\tau_{\mathrm{PR}}$ for micron-sized or larger particles. $\tau_{\mathrm{PR}}$ equals $\tau_{\mathrm{E}}$ at a particle mass of about $10^{-15} \mathrm{~kg}$. For the largest asteroids, $\tau_{\mathrm{cc}}$ is of the order of $10^{8}-10^{9} \mathrm{yr}$; these asteroids therefore have somewhat longer but comparable lifetimes to the Apollo asteroids (Öpik, 1963).

We mention, in conclusion, that our collisional model is consistent with observation whenever a comparison is possible. The theoretical population index is within the standard deviation of the empirical one. The lifetimes of the large objects compare reasonably with the lifetime of Apollo asteroids estimated by Öpik. The erosion rate of large objects is not unreasonable in view of the fact that lunar highlands are saturated by craters of the order of many kilometers in diameter. An order-of-magnitude statistical treatment of the top three masses indicates that practically all other asteroids and their fragments in the asteroidal belt are in steady-state condition and the present collisional model appears justified.

\section{References}

Dohnanyi, J.S. (1966)

Dohnanyi, J.S. (1967a)

Dohnanyi, J.S. (1967b) (to be published).

Dohnanyi, J.S. (1967c)

Gault, D.E., Shoemak

Hawkins, G.S. (1960)

Hawkins, G. S. Upton E. K. L. (1958)

Kaiser, T.R. (1961) Monthly Not. R. astr. Soc., 123, 265.

Kuiper, G.P., Fujita, Y., Gehrels T., Groeneveld, I., Kent, J., Van Biesbroek, G., Van Houten, C.J. (1958) Astrophys. J., Suppl. Ser., 3, 289.

Narin, F.J. (1966) Spacecraft, 3, 1438.

Öpik, E.J. (1963) Adv. Astr. Astrophys., 2, 219.

Robertson, H.P. (1936) Mon. Not. R. astr. Soc., 97, 423.

Southworth, R.B.(1967) Int. Symp. on the Zodiacal Light and the Interplanetary Medium, Honolulu (to be published).

Whipple, F. L. (1963) Smithson. Contr. Astrophys., 7, 239.

\section{DISCUSSION}

Whipple: I wish to congratulate Dr. Dohnanyi for this solution to a long-standing important problem. Piotrowski attempted such a solution some 20 years ago without obtaining a definitive answer, although his approximate solution was not far from Dohnanyi's.

Dohnanyi: I am in debt to Piotrowski for some of the ideas I used in this analysis, but I regret to say that I disagree with the formulation of Piotrowski's analysis (regarding fragmentation during collisions). 
Sekanina: What considerations have lead you to the accepted average collision velocity of $5 \mathrm{~km} / \mathrm{sec}$ ? Dohnanyi: The distribution of asteroids in inclination and eccentricity as given by Watson.

Bronsten: Have you used the average velocity of collisions $(5 \mathrm{~km} / \mathrm{sec})$ only, or have you taken into account the velocity distribution?

Dohnanyi: I have only used the average velocity of $5 \mathrm{~km} / \mathrm{sec}$. The distribution could be described, approximately, by a collisional velocity of $5 \pm 5 \mathrm{~km} / \mathrm{sec}$. Since, however, the steady-state value of the index is rather insensitive to the velocity, I do not feel there is a danger of substantial error involved in using a simple collisional velocity rather than a distribution in the present simplified model.

Delcourt: From the very beginning, you have taken a particular form (power law) for the massdensity function as steady-state solution. Can you find some other solution?

Dohnanyi: I do not know if another solution exists; I have not investigated the uniqueness of my solution.

Delcourt: You consider a homogeneous system. Will you extend your model?

Dohnanyi: My model contains spherical objects only with identical material properties (exclusive of size).

Sekanina: Would your model make it possible to estimate the probable age of the asteroid belt?

Dohnanyi: I do not think so; the statistical treatment employed in the present model includes 'smoothing' over of the details of physical processes and it is difficult to reverse such a calculation in order to obtain unique initial conditions. 